\title{
The research of traffic flow in LaoTieshan channel based on AIS data
}

\author{
Qiang Tu ${ }^{1, a}$ and Zhongyi Zheng ${ }^{1}$ \\ ${ }^{1}$ Dalian Maritime University, Navigation college, Dalian, Liaoning Province, 116000, China
}

\begin{abstract}
In order to study the ship traffic flow in LaoTieshan channel, this paper rewrites the rules of NS (Nagel-Schreckenberg) model of Cellular Automata (CA) based on AIS (Automatic Identification System) data. By referring to the idea of synchronous flow in three-phase traffic flow theory and introducing the speed adaptation mechanism into the simulation, the new model reproduces the traffic flow of LaoTieshan channel and gets the relevant fundamental diagrams. Compared with the actual flow-density diagram drawn from AIS data, it is found that the simulated flow generated by the improved model is more in line with the actual condition, and also conforms to the phase transition of traffic flow in LaoTieshan channel. The method and idea of the simulation are helpful to explain and investigate some complex situations in LaoTieshan channel.
\end{abstract}

\section{INTRODUCTION}

As a research method of complexity science, Cellular Automata (CA) is not only widely used in the research of physical phenomena, road traffic and so on, but also increasingly used in the research of traffic flow in busy waterways. Hongxiang Feng [1] rewrites the rules of NS model based on AIS data, so as to analyze the three-phase change of traffic flow in channels; Qiang Meng [2] proposes a discrete model based on the Cellular Automata model by analyzing the characteristics of the Singapore Strait according to the subjective decisions of the ship pilot; Le Qi [3] discovers the possibility of "pseudo lane change" when studying the narrow channel, so he puts forward a new Cellular Automata model based on space-logic rules. All of the above studies are based on the analysis of AIS data to find the characteristics of ships or channels in the area of research to establish a new model. It can be seen that the AIS data plays a fundamental and important role in the research of marine traffic engineering, especially in marine traffic flow.

\section{The processing of AIS data}

\subsection{Rule of ship arrival}

We set a threshold at the entrance of LaoTieshan channel in the southeast to northwest route, and statistics of the number of ships arriving every day for 90 consecutive days from June to August are used to calculate the arrival rules of ships [4][5]. By comparing the nuclear density curve with the theoretical Poisson distribution curve, it can be seen that the rule of ship arrival roughly obeys the Poisson distribution (Figure 1). In order to ensure the accuracy, we carry out KS TEST (Kolmogorov Smirnov test). Through the test value $H=0, s=0.568$, it can be confirmed that the data source conforms to Poisson distribution.

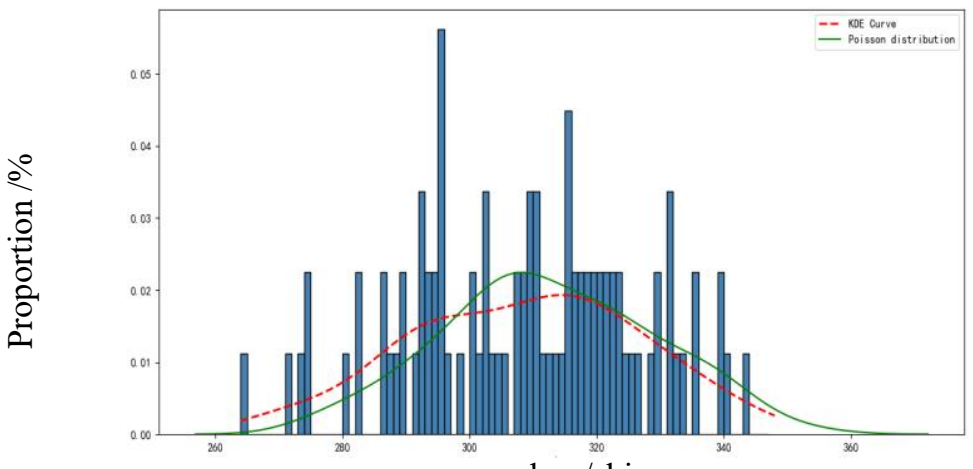

number /ships

Figure 1.The statistics of ship arrival rule in the southeast to northwest route

a Corresponding author: icetamia@sina.com 


\subsection{The statistics of ship speed and length of ships}

Through the processing of AIS data, we drew the nuclear density curve of the speed and length distribution in 7 days compared with the standard Normal distribution curve, and it is found that the overall ship speed distribution tends to be Normal distribution; the KS test can't get the satisfactory results due to the huge amounts of speed data in 7 days , the Quantitle-Quantile (Q-Q) test map is chosen to deal with the data. When the distribution concentrates on the Normal distribution straight line, it shows that this set of data obeys the Normal distribution (Figure $2 \& 3$ ).

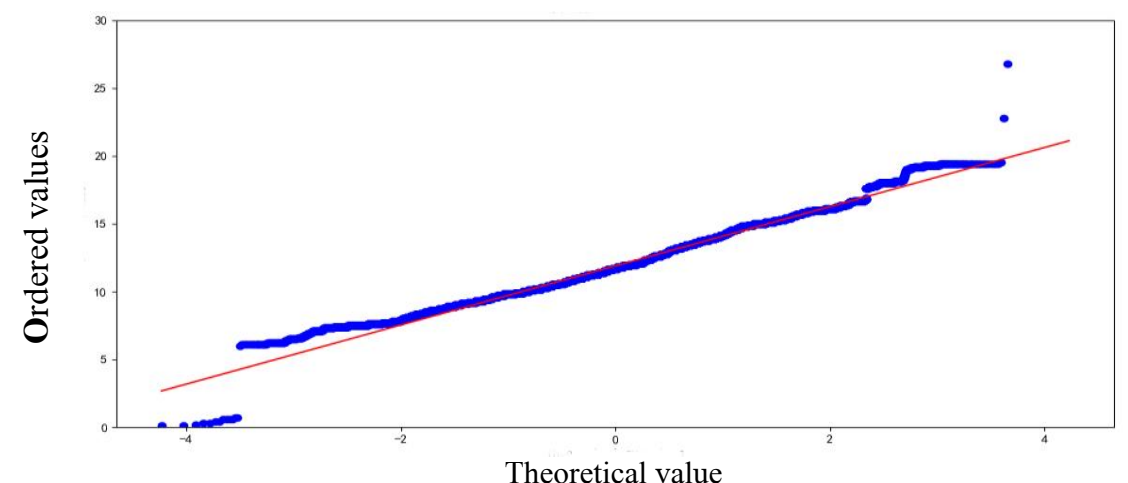

Figure 2.The Q-Q test map of ship speed

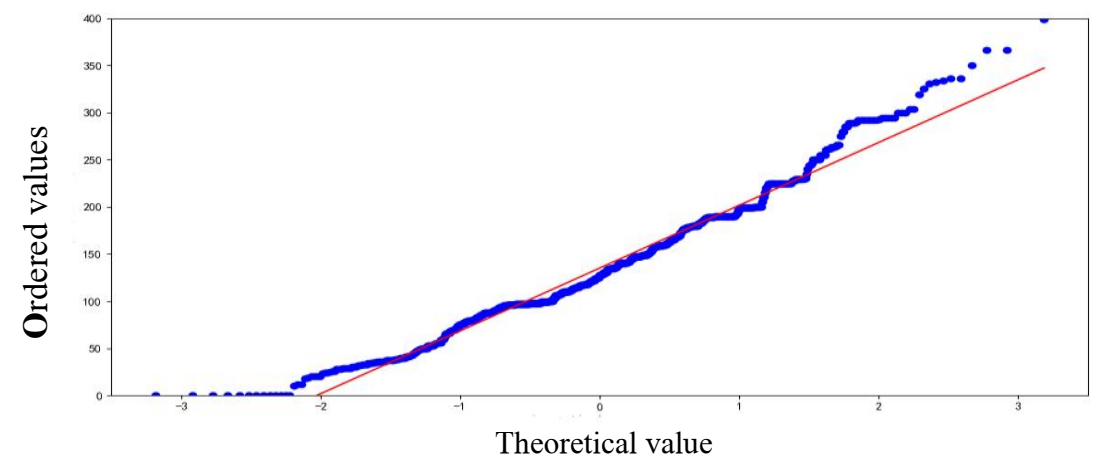

Figure 3.The Q-Q test map of length of the ships

\section{Simulation and analysis}

\subsection{Improved NS cellular automata model with speed adaption mechanism}

In this paper, without considering the dispute between the two kinds of traffic flow theories, we rewrite from the rationality of the two theories, and apply this idea to the research of marine traffic engineering. The idea of synchronization has its rationality whether from the perspective of energy consumption or the pilot's custom. In view of this, we introduce the speed synchronous adaptation mechanism, the core of the three-phase traffic flow theory, into the NS model under the fundamental diagram theory. So we use the reasonable part of the three-phase traffic theory as a reference and build an improved version of the NS model to simulate the traffic flow in LaoTieshan channel. The rules are as follows:

$$
\begin{aligned}
& \text { (1) acceleration: } V_{(i, t+1)}=\min \left(V_{(i, t)}+1, V_{\max }\right) \\
& \text { (2) deceleration: } V_{(i, t+1)}=\min \left(V_{(i, t+1)}+1, d_{(i, t)}+V_{(i+1, t+1)}^{\prime}\right) \\
& \text { while: } V_{(i+1, t+1)}^{\prime}=\min \left(V_{\max }-1, V_{(i+1, t)}, \max \left(0, d_{(i+1, t)}-1\right)\right) ; \\
& \text { (3) random slow with probability } p: V_{(i, t+1)}=\max \left(V_{(i, t+1)}-1,0\right) \\
& \text { while: } P=\left\{\begin{array}{ll}
P_{1} & d_{(i, t)}+V_{(i+1, t+1)}^{\prime}>d_{0} \\
P_{2} & d_{(i, t)}+V_{(i+1, t+1)}^{\prime}<d_{0}
\end{array} ;\right. \\
& \text { (4) movement of position: } \quad X_{(i, t+1)}=X_{(i, t)}+V_{(i, t+1)} .
\end{aligned}
$$

Where $V_{(i, t)}$ is the speed of the $\mathrm{i}$ ship at $\mathrm{t}$ time step, $X_{(i, t)}$ represents the position of the $\mathrm{i}$ ship at $\mathrm{t}$ time step, and $d_{(i, t)}$ is the space distance between the $\mathrm{i}$ ship and the front ship at $\mathrm{t}$ time step, i.e. the numbers of empty cells and $p_{1}, p_{2}, d_{0}, V_{\max }$ are constants. The slow probability $p$ is no longer a constant, but will change with the distance between the front and rear ship, coupled with the variation of the simulated speed of the front ship in the new model, which is the result of introducing the speed adaptation mechanism. That is to say, the new model is an improved NS model considering the change of slow probability and the speed of the front ship 


\subsection{The parameters of cells}

The length of the southeast to northwest route is $9 \mathrm{n}$ mile, about $16668 \mathrm{~m}$. As the length of ships obeys the Normal distribution, we choose the mean value of Normal distribution $150 \mathrm{~m}$ as the simulated length in order to facilitate the simulation and the speed varies from 6 to $16 \mathrm{kn}$, conforming to the Normal distribution [6]. The ships in the channel are set to sail in the rule of following, and overtaking is not allowed.

\subsection{The comparison of simulation and mechanism analysis}

\subsubsection{The comparison of the two models}

According to the above parameters and data, in the NS model, the slow probability is set to be 0.3 , $L$ waterway $=556$ cells, $V \max =16 \mathrm{kn}$. With other conditions remained unchanged, by introducing the speed adaptation mechanism, the improved model can adjust itself according to the degree of congestion under the new boundary conditions. The slow probability is set to be $P 1=0.1, P 2=0.5$ through the observation of AIS data. The total time steps are 1400 [7].

Since the two kinds of simulation are essentially carried out under the framework of the fundamental diagram theory, we put the fundamental diagrams of the two kinds of simulation together (Figure 4).

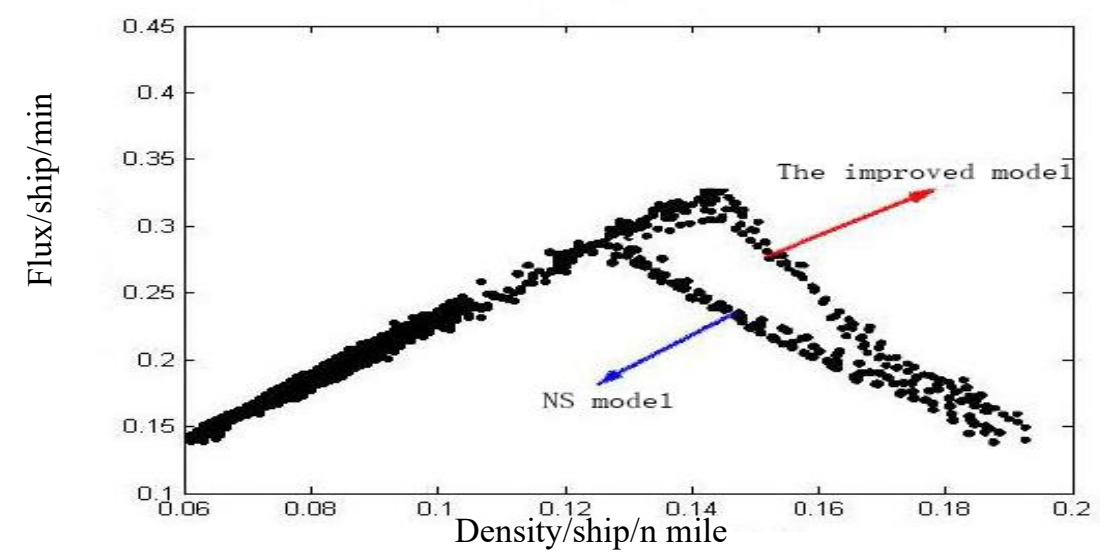

Figure 4. The comparison diagram of the two models

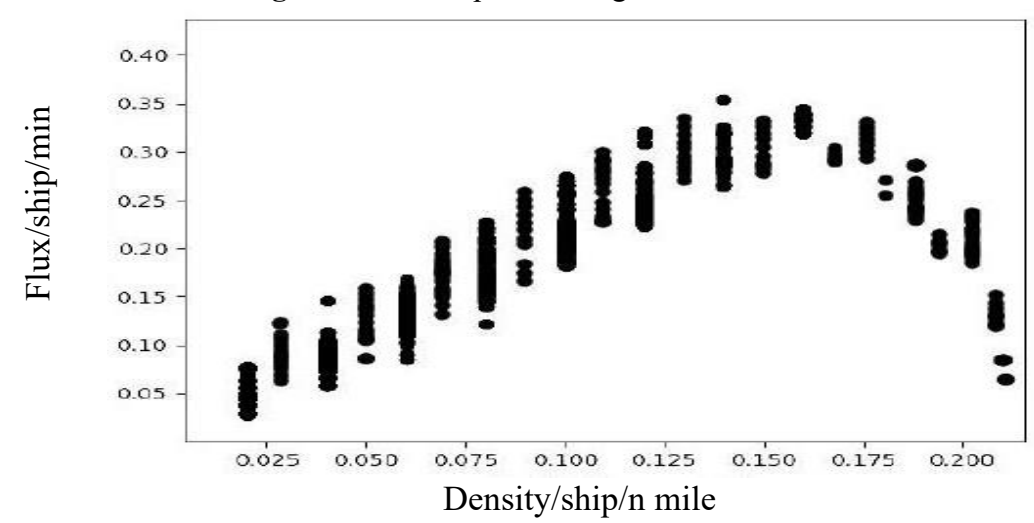

Figure 5.The flow-density diagram drawn from AIS data

\subsubsection{Comparison of simulated and measured diagrams}

According to formula: $Q=\rho \cdot \bar{v} \cdot w$, $\rho=\frac{N}{L \cdot w}$ and $\bar{v}=\frac{1}{n} \sum_{i=1}^{n} v_{i}[8]$, the flow-density diagram of the measured AIS data is processed and drew as follows:
As the measured diagram is drawn by a large number of discrete points of data, so we average the data points and redraw them into a comparison chart compared with the two kinds of simulation diagrams (Figure 6). 


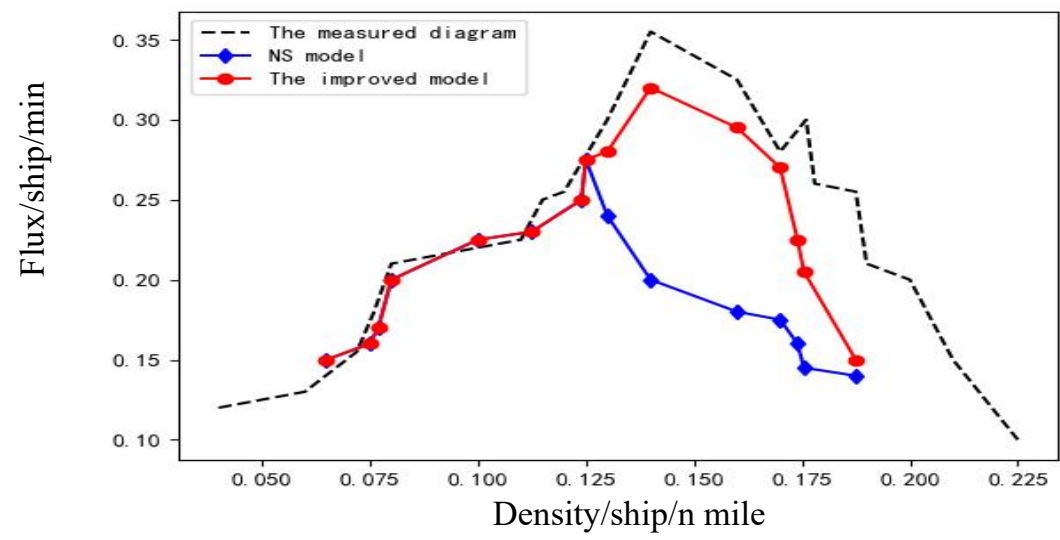

Figure 6. The comparison chart

\subsubsection{Analysis of the measured diagram}

From the perspective of traffic flow theory, the improved model with speed adaption mechanism has a better stability compared with NS model, so it can nicely simulate the real situation of traffic flow [9][10]. The introduction of speed adaption mechanism can automatically dissipate the congestion in the channel due to the random disturbance before the maximum value of flow is reached, avoiding the occurrence of behavior of over-deceleration, and prevent the whole channel from entering into the jam in advance, so that the density and flux of the traffic flow could increase relatively smoothly instead of fluctuating growth. Employing the improved model to obtain the fundamental diagram and the space-time diagram could be used to acquire the maximum value of the traffic flow, the density of jam and the speed of accumulation and dissipation wave, which is of certain significance for the study of traffic flow in LaoTieshan channel and the management for MSA.

\section{CONCLUSION}

(1) Considering the speed of the front ship breaks the rule that the front ship is regarded as a static cell in NS model ,namely, the change of the relative speed, and through the introducing of speed adaption mechanism, we set up a varing slow probability, which makes the ship speed up to catch up when the distance between the ship and the front ship increases as well as to slow down to keep distance when it is less than the safe distance.

(2) This paper is designed to model the situation of following, so there are some differences compared with the actual measured diagram, indicating that there are still some deficiencies in the internal operation mechanism.

\section{References}

1. Hongxiang Feng, Fanyou Kong, Yingjie Xiao, Xiaojun Yang Analysis of characteristic parameters of ship traffic flow of cellular automaton model based on AIS [J]. Journal of
Wuhan university of technology (traffic science and engineering), 2014, 38(02):324-328.

2. Xiaobo Qu, Qiang Meng. Development and applications of a simulation model for vessels in the Singapore Straits[J].Expert Systems with Applications. 2012, 39(9):8430-8438.

3. Le QI, Zhongyi Zheng, Longhui Gang. A cellular automaton model for ship traffic flow in waterways[J].Physica A:Statistical Mechanics and its Applications.2017, 471:705-717.

4. Yunda Xia. Study on the complex characteristics of road traffic flow based on cellular automaton model [D] Hunan University, 2018.

5. Boris S Kerner, Sergey L Klenov, Dietrich E Wolf. Cellular automata approach to three-phase traffic theory $[\mathrm{J}]$. Journal of Physics A:Mathematical and General.2002, 35(47):9971.

6. Kun Gao. From fundamental diagram theory to three-phase traffic flow theory [D] University of Science and Technology of China, 2008.

7. Jingxian Liu. Feng Zhou, Maoqing Wang. Simulation of waterway traffic flow at harbor based on the ship behavior and Cellular Automaton, 2010[C].2010 International Conference on Artificial Intelligence and Computational Intelligence, 23-24 Oct 2010.

8. Zhaolin $\mathrm{Wu}$, Jun Zhu. Marine Traffic Engineering [M].Dalian Maritime University Press. 2004.

9. Jiangcen Ke. Research on transportation organization of water transportation hub based on cellular automaton [D]. Wuhan university of technology, 2012.

10. Xingang LI, Ziyou GAO, Bin JIA, Rui JIANG. Deceleration in advance in the Nagel-Schreckenberg traffic flow model [J].Physica A:Statistical Mechanics and its Applications.2009, 388(10):2051-2060. 